\title{
Morphological Multiscale Stationary Wavelet Transform based Texture Segmentation
}

\author{
Mosiganti Joseph Prakash \\ Professor, CSE Dept., School of Engineering and Technology, GNITC, Hyderabad, A.P, INDIA \\ E-mail: mjosephp7@gmail.com \\ Dr.Kezia.J.M \\ Professor, ECE Dept, School of Engineering and Technology, GNITC, Hyderabad, A.P, INDIA \\ E-mail: sakakezia1981@gmail.com \\ Dr.V.Vijaya Kumar \\ Professor \& Dean of CSE\&IT Depts., Anurag Group of Institutions, Hyderabad, A.P, INDIA \\ E-mail: vijayvakula@yahoo.com
}

\begin{abstract}
Image segmentation is an important step in several computer vision applications. The segmentation of images into homogeneous and meaningful regions is a fundamental technique for image analysis. Textures occupy a vital role in a wide range of computer vision research fields; from microscopic images to images sent down to earth by satellites, from the analysis of multispectral scan images to outdoor scenes, all consist of texture. Although several methods have been proposed, less work has been done in developing suitable techniques for segmentation of texture images. After a careful and in-depth survey on wavelet transforms, the present study found that efficient numerical solutions in the signal processing applications can be found using Stationary Wavelet Transform (SWT). SWT is redundant, linear and shift invariant, that's why it gives a better approximation than the DWT. In this paper a novel texture segmentation method based on "SWT and Textural Properties" is proposed. Multi scale SWT with Textural Properties and morphological treatment is used in the present study to detect fine edges from texture images for a fine segmentation.
\end{abstract}

Index Terms - SWT, texture, segmentation, morphology.

\section{INTRODUCTION}

One of the first and most important operations in image analysis and computer vision is segmentation. The aim of image segmentation is the domain independent partition of the image into a set of regions, which are visually distinct and uniform with respect to certain properties, such as grey level, texture or color. The problem of segmentation is an important research field and many segmentation methods have been proposed in the literature. Many segmentation methods are based on two basic properties of pixels in relation to their local neighborhood: similarity and discontinuity. Pixel similarity gives rise to region-based methods, whereas pixel discontinuity gives rise to boundary-based methods.

Image segmentation is an important research area in computer vision and hundreds of segmentation algorithms have been proposed in the last 30 years. Boundary based or region-based methods alone often fail to produce accurate segmentation results [1]. In fact, color and texture are fundamental features in defining human visual perception. Hence, complementary information such as brightness, color and texture is taken into consideration $[2,3,4]$.

Image segmentation based on texture is to partition an image into homogeneous regions and identify the boundaries which separate regions of different texture. One of the widely used segmentation methods is to apply filters on image and calculate the gradient in texture feature space. Based on the observation that only several distinct filter responses are needed to represent textures, Malik etc. [5, 6] group the filter responses into a small set of prototype response vectors which are called "textons". Thereafter, they developed a texture gradient algorithm using these textons to generate a soft boundary map for image segmentation.

Texture is defined as something consisting of mutually related elements. A texture may be fine, coarse, smooth, or grained depending upon its tone and structure. While tone is based on pixel intensity properties, structure is the spatial relationship between pixels [7]. In case of statistical approach, texture is defined by a set of statistically extracted features represented as vector in multidimensional feature space. The statistical features could be based on first-order, second-order or higherorder statistics of gray level of an image. Texture based methods are best suited for segmentation of medical image, when compared to segmentation of medical image using simple gray level based methods [8-10]. Texturebased medical images include mammograms [11, 12], ultrasonic liver images [13-15], and X-Ray lung images $[16,17]$. Edge based image analysis usually implies 
assumption that the local intensities are uniform in pixel belonging to the same object which is typically not the case in grayscale medical images [18]. Texture-based image analysis is not limited by this assumption. However, it has been proposed that texture can be characterized by relatively small scale structures which are distributed uncertainly relative to the object [19]. It is apparent that these characteristics are typical of variances in medical images.

Texture-based image segmentation is a fundamental task for computer vision. The existing methods for texture based segmentation are model based methods [2027] and multi-resolution multi-channel methods [28-31].

In order to achieve the objective of designing effective algorithms which could provide the properties pointed out by Haralick [32], the ingredients essential for a textured image segmentation system are:

- Set of texture features having good discriminating power;

- Segmentation algorithm having spatial constraints;

- Estimation of texture features taking the nature of the feature image planes into account. Selection of texture features from a set of existing texture features [33-43] which can provide good discriminating power and are easy to compute to serve the need is very important.

The paper is organized as follows. In section I brief introduction, in section II Texture, section III deals with stationary wavelet transform, section IV deals with Advantages of SWT in image processing applications, in section $\mathrm{V}$ proposed methodology, results \& discussions are given in section VI and finally conclusions are given in section VII.

\section{TEXTURE}

Texture plays an important role in many machine vision tasks such as surface inspection, scene classification, and surface orientation and shape determination. For example, surface texture features are used in the inspection of semiconductor wafers, graylevel distribution features of homogeneous textured regions are used in the classification of aerial imagery, and variations in texture patterns due to perspective projection are used to determine three dimensional shapes of objects. Texture is characterized by the spatial distribution of gray levels in a neighborhood. Thus, texture cannot be defined for a point. The resolution at which an image is observed determines the scale at which the texture is perceived. For example, in observing an image of a tiled floor from a large distance we observe the texture formed by the placement of tiles, but the patterns within the tiles are not perceived. When the same scene is observed from a closer distance, so that only a few tiles are within the field of view, we begin to perceive the texture formed by the placement of detailed patterns composing each tile. For our purposes, we can define texture as repeating patterns of local variations in image intensity which are too fine to be distinguished as separate objects at the observed resolution. Thus, a connected set of pixels satisfying a given gray-level property which occurs repeatedly in an image region constitutes a textured region. A simple example is a repeated pattern of dots on a white background. Text printed on white paper such as this page also constitutes texture. Here, each gray-level primitive is formed by the connected set of pixels representing each character. The process of placing the characters on lines and placing lines in turn as elements of the page results in an ordered texture.

Before either segmentation or classification can take place, some homogeneity or similarity criterion must be defined. These criteria are normally specified in terms of a set of feature measures, which each provide a quantitative measure of a certain texture characteristic. These feature measures are alternatively referred to here as texture measures or just simply features. Simple measures of texture may be derived based upon the moments of the gray-level probability distribution function (PDF) of the given image. The $\mathrm{k}^{\text {th }}$ central moment of the PDF $p(l)$ is defined as

$$
m_{k}=\sum_{l=0}^{L-1}\left(l-\mu_{f}\right)^{k} p(l)
$$

Where $l=0,1,2, \ldots \ldots, L-1$ are the gray levels in the image $\mathrm{f}$, and $\mu_{f}$ is the mean gray level of the image given by

$$
\mu_{f}=\sum_{l=0}^{L-1} l p(l)
$$

The second central moment, which is the variance of the gray levels is given by

$$
\sigma_{f}^{2}=m_{2}=\sum_{l=0}^{L-1}\left(l-\mu_{f}\right)^{2} p(l)
$$

The normalized third moment which is known as skewness is given as

$$
\text { skewness }=\frac{m_{3}}{m_{2}^{3 / 2}}
$$

Skewness indicate the asymmetry of the probability distribution function. Since skewness represent the discrepancies in the pixels of a given image it is considered as the important texture feature for the image segmentation.

\section{STATIONARY WAVELET TRANSFORM}

The SWT is independently developed by several researchers and under different names, e.g. the undecimated wavelet transforms, the invariant wavelet transform and the redundant wavelet transform. This algorithm is more famously called by Holdschneider et al., [44] as "algorithme à trous" in French (word trous means holes in English) which refers to inserting zeros in the filters. 
The SWT provides efficient numerical solutions in the signal processing applications. It gives a better approximation than the DWT, since it is redundant, linear and shift invariant. These properties allow SWT to be realized using a recursive algorithm but experiences the drawback of very high redundancy and involved computations. In SWT, the major problem of translationinvariance experienced by DWT is overcome by removing the down samplers and up samplers in the DWT and up sampling the filter coefficients by a factor of $2^{(j-1)}$ in the $j^{\text {th }}$ level of the algorithm.

The SWT is an inherently redundant scheme as the output of each level of SWT contains the same number of samples as the input, so for a decomposition of $\mathrm{N}$ levels there is a redundancy of $\mathrm{N}$ in the wavelet coefficients. Fig.1 details the digital implementation of SWT up to level 3 coefficients. From the diagram we could see that, the filters in each level are up-sampled versions of the previous. Fig. 2 illustrates the SWT decomposition tree and wavelet decomposition tree for 3 levels. In the computation of the SWT of a signal $x(k), W_{j k}$, and $V_{j k}$ are the detail and the approximation coefficients of the SWT. The filters $H_{j}$ and $G_{j}$ are the standard low pass and high pass wavelet filters, respectively. In the first step, the filters $H_{1}$ and $G_{1}$ are obtained by up sampling the filters using the previous step (i.e. $H_{j}-1$ and $G_{j}-1$ ).
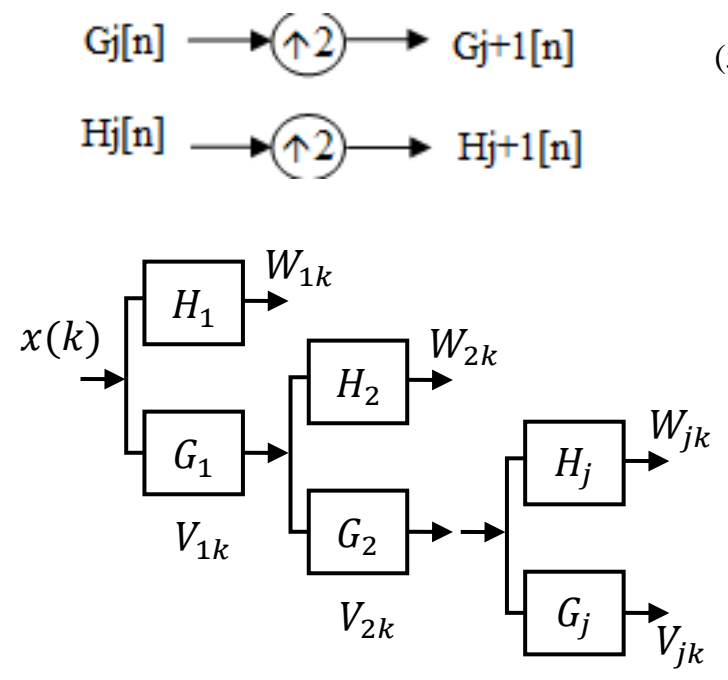

Fig 1. A 3-level SWT digital implementation

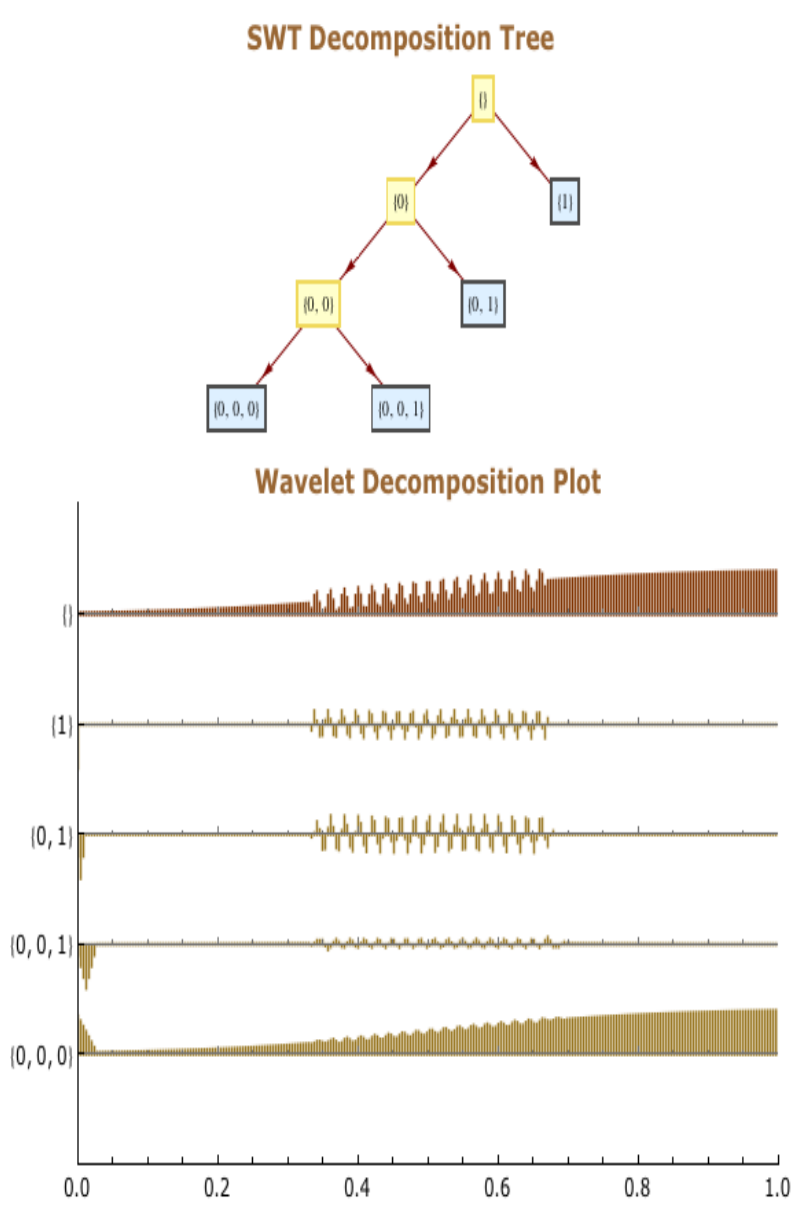

Fig 2. SWT Decomposition tree and wavelet decomposition plot

\section{AdVANTAGES OF SWT IN IMAGE PROCESSING APPLICATIONS}

The major problem of translation shift invariance experienced by DWT is overcome by SWT and it provides efficient numerical solutions in the signal processing applications. Moreover SWT gives a better approximation than the DWT, since it is redundant, linear and shift invariant. These properties allow SWT to be realized using a recursive algorithm but experiences the drawback of very high redundancy and involved computations. 
1. SWT has an evident advantage over DWT when the requirement of real time image processing applications are not high, and it can offer more precise flow information.

2. SWT is translation invariant, even if the signal is shifted, the obtained coefficients will not change which happens to be the highly desired property for many image processing applications, edge detection, image fusion and break down point detection.

3. DWT is only suitable for implementing for discrete signals of images whose size is a power of 2 but SWT can be applied to any arbitrary size of images.
4. In few harmonic analyses, energy levels and phase information are required. The advantage of SWT is that it does not require the above information. The time of occurrence and type of disturbances of SWT is calculated directly from the detail coefficients.

\section{PROPOSED METHODOLOGY}

The proposed method is as given below and it consists of 9 steps. the block diagram for the entire process is given in Fig. 3.
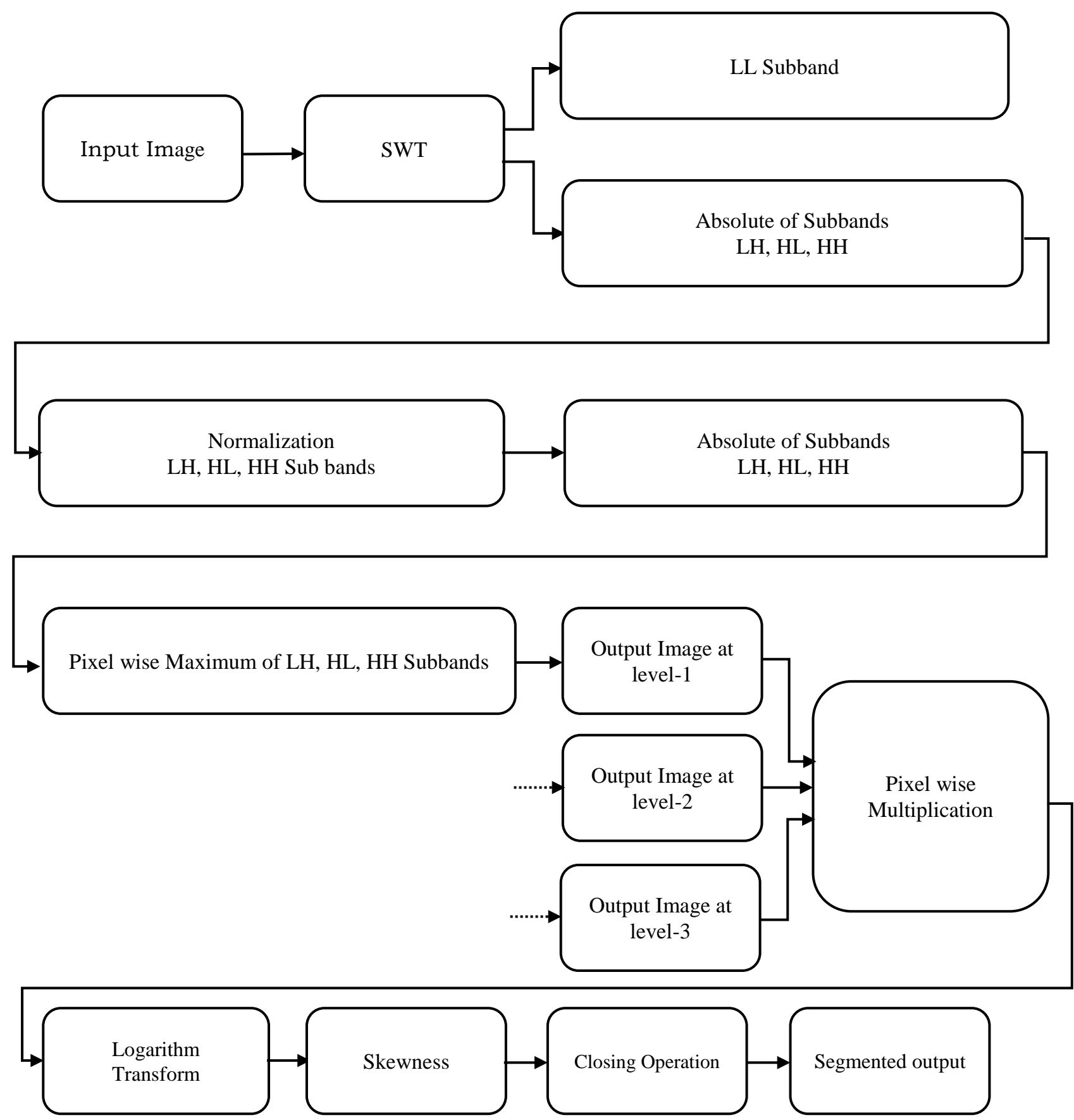

Fig 3. Block diagram of proposed algorithm 


\section{Alogorithm Steps}

First step: Stationary wavelet transform (SWT) is applied on the input image to obtain the translation invariant property, and also to gain other advantages as specified in section 4.1 In the first level of SWT - LL1, LH1, HL1, HH1 are formed.

Second step: To eliminate complex values absolute value is calculated for horizontal, vertical and diagonal details in step two.

Third step: The output images of step two are normalized to the maximum values of corresponding images.

Fourth step: in step four again absolute values are calculated for the output images of step three and pixel wise maximum is taken for all the images. The obtained image is named as "output image at level-1".

Fifth step: In the same way on LL1 sub band of step one SWT is applied. By this second level of SWT - LL2, LH2, HL2, HH2 are formed. On this - step two to four are repeated to form "output image at level-2". The same process is repeated on LL2 and "output image at level-3" is obtained.

Sixth step: pixel wise product is calculated for all the maxims of output image 1, 2 and 3.

Seventh step: logarithmic transform is applied to reduce the noise levels in the output of sixth step.

Eighth step: Skew-ness represents the discrepancies in the pixels of a given image, that's why it is considered as the important texture feature for the image segmentation. In the present work skewness is calculated in overlapping $5 \times 5$ blocks in the eigth step as given in equation 4 .

Ninth step: Finally morphological closing operation is applied in order to achieve further noise reduction and to fill small holes.

\section{EXPERIMENT RESULTS AND DISCUSSION}

The proposed segmentation scheme based on SWT-TP is tested on bark textures, stone and fabric textures of vistex and brodatz databases.

The output after the pixel wise product of first level and second level of SWT maxima is shown in Fig. 4 (b), 5 (b) \& 6(b). The output after the product of first level, second and third level of SWT maxima is shown in Fig. 4 (c), 5(c) \& 6(c). Fine edges are visible in Fig. 4(c), 5(c) \& 6(c) when compared to Fig. 4 (b), 5 (b) \& 6(b). The skew-ness image in Fig. 1(d), 2(d) \& 3(d) show clearly the discrepancies in the pixels, and thus results a fine segmentation. In order to eliminate tiny holes and for further noise reduction finally morphological closing operation is performed, the output of which is shown in Fig. 4(e), 5(e) \& 6(e).

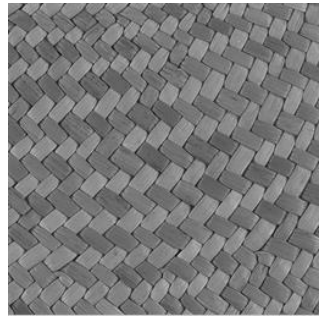

(a)
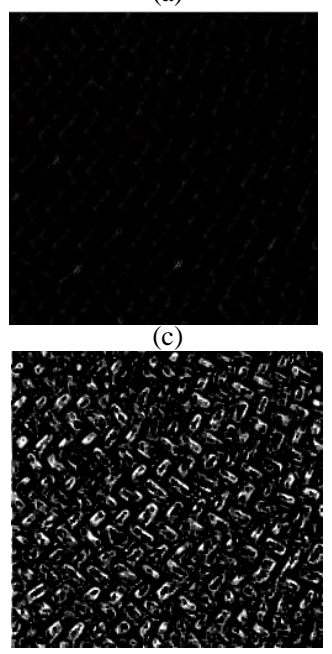

(e)

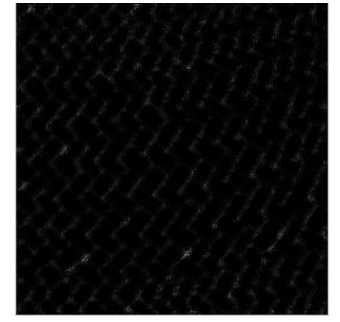

(b)

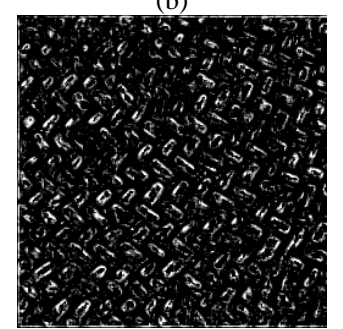

(d)
Fig 4. Fabric 0 image segmentation experimental results (a) Original image (b) Product of two maximas (c) Product of three maximas (d) Skewness (e) Closing

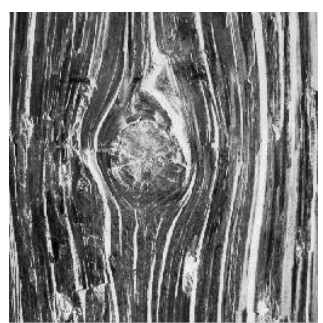

(a)

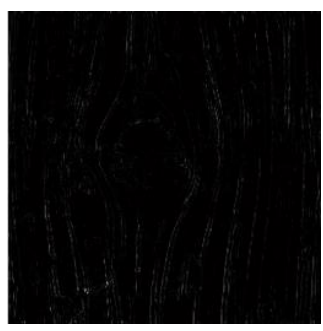

(c)

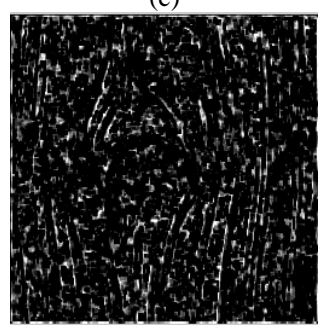

(e)

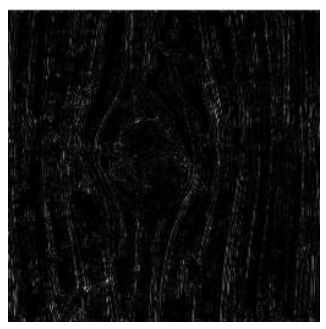

(b)

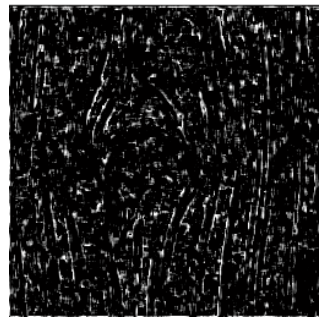

(d)
Fig 5. D72 image segmentation experimental results (a) Original image (b) Product of two maximas (c) Product of three maximas (d) Skewness (e) Closing 


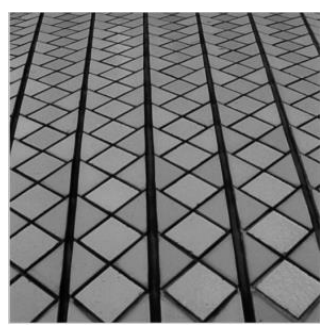

(a)

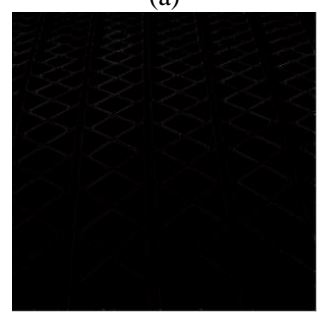

(c)

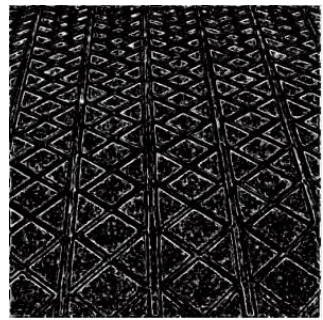

(e)

Fig 6. image segmentation experimental results (a) Original image Tile 0 (b) Product of two maximas (c) Product of three maximas (d) Skewness (e) Closing

\section{CONCLUSION}

The proposed segmentation algorithm based on SWT and textural properties with morphological treatment obtained the information on the asymmetry of the pixels in the image, thus the algorithm is able to segment the image very effectively. The proposed method is efficient in reducing the noisy effects in the image and is also able to eliminate information loss by the usage of logarithmic transform and SWT respectively. The proposed algorithm does not make use of any edge detection operator for segmentation; therefore the method overcomes the edge detection operator's disadvantage of noise enhancement.

The first consideration of the proposed segmentation scheme based on SWT and textural properties with morphological treatment is its simplicity. The proposed technique is simple, and its computational cost is low. It is an iterative process just requiring the following two operations per iteration: the application of a single iteration of the SWT and the evaluation of the pixel wise maxima. Moreover, no profiteering step is added, and the method is not dependent on the statistics of the input image. Nevertheless, at first sight, the most noticeable effect of the proposed technique is the contrast achieved between edges and background, which is, in fact, the main objective of any edge enhancement algorithm. The adaptation capability of the method it does not require any prior knowledge of the image.

\section{ACKNOWLEDGMENT}

The authors would like to thank Sardar Tavinder Singh Kohli Chairman, Sardar Gagandeep Singh Kohli Vice Chairman and Dr H. S. Saini Managing Director, Guru Nanak Technical Institutions for their encouragement towards research activities. Authors extended their gratitude to Management of Anurag Group of Institutions for providing necessary infrastructure for Centre for Advanced Computing Research (CACR) at AGOI, which is bringing various research scholars across the nation to work under one roof. The CACR is providing a research platform for exchanging and discussing various views on different research topics in CSE.

Authors would like to thank anonymous reviewers for their valuable comments. The authors would like to express their gratitude to Dr.G.Tulsi Ram Das, Vice chancellor, JNTU Kakinada.

\section{REFERENCES}

[1] Jordi Freixenet, Xavier Muñoz, Joan Martí, Xavier Lladó, "Color texture segmentation by region-boundary cooperation", European Conference on Computer Vision, 2004.

[2] Jitendra Malik, Serge Belongie, Thomas Leung, Jianbo Shi, Contour and Texture Analysis for Image Segmentation", International Journal of Computer Vision 43(1), 7-27, 2001.

[3] J. Freixenet, X. Munoz, D. Raba, J. Mart'1, and X. Cuf, Yet Another Survey on Image Segmentation:Region and Boundary Information Integration”, in ECCV, 2002.

[4] Morten Rufus Blas, Motilal Agrawal, Aravind Sundaresan, Kurt Konolige, "Fast texture Color/segmentation for outdoor robots", 2008 IEEE/RSJ International Conference on Intelligent Robots and Systems ,Nice, France, Sept, 2226, 2008.

[5] J. Malik, S. Belongie, T. K. Leung, and J. Shi., "Contour and texture analysis for image segmentation", International Journal of Computer Vision, 43(1):7-27, 2001.

[6] D. Martin, C. Fowlkes, and J. Malik, "Learning to detect image boundaries using brightness and texture", In Proceedings of NIPS, pages 1255-1262, 2002.

[7] T. Pavlidis and Y. Liow, "Integrating region growing and edge detection", IEEE Transactions on Pattern Analysis and Machine Intelligence, pages 225-233, 1990.

[8] Saka Kezia, Dr.I.Shanti Prabha, Dr.V.Vijayakuamr, "A New Texture Segmentation Approach for Medical Images", International Journal of Scientific \& Engineering Research, Vol. 4, No. 1, pp.1-5, January 2013.

[9] Saka Kezia, Dr.V.VijayaKumar, Dr.I.Santi Prabha, "Auto Detection of Tubercle Bacilli Based on Wavelets", International Journal on Graphics Vision and Image Processing, Vol. 11, No. 3, pp.980-986, June 2011.

[10] Saka Kezia, Dr.I.Santi Prabha, Dr.V.VijayaKumar, "Innovative Segmentation Approach Based on LRTM", International Journal of Soft Computing and Engineering, Vol. 2, No. 5, pp. 229-233, November 2012.

[11] Xu S, Liu H, Song E, "Marker-controlled watershed for lesion segmentation in mammograms", Journal of Digit Imaging. 2011 Oct; 24(5):754-63. 
[12] Oliver A, Lladó X, Pérez E, Pont J, Denton ER, Freixenet J, Martí J, "A statistical approach for breast density segmentation", J Digit Imaging. 2010 Oct; 23(5):527-37.

[13] Pradeep Kumar B.P, Prathap.C, Dharshith.C.N, "An Automatic Approach For Segmentation of Ultrasound Liver Images", IJETAE, Volume 3, Issue 1, January 2013.

[14] Cao G, Shi P, Hu B., "Liver fibrosis identification based on ultrasound images", Conf Proc IEEE Eng Med Biol Soc. 2005;6:6317-20.

[15] Li G, Luo Y, Deng W, Xu X, Liu A, Song E, “Computer aided diagnosis of fatty liver ultrasonic images based on support vector machine", Conf Proc IEEE Eng Med Biol Soc. 2008.

[16] Hassen DB, Taleb H., "Automatic detection of lesions in lung regions that are segmented using spatial relations", Clin Imaging. 2013 May-Jun; 37(3):498-503.

[17] Tan Y, Schwartz LH, Zhao B., "Segmentation of lung lesions on CT scans using watershed, active contours, and Markov random field", Med Phys. 2013 Apr; 40(4).

[18] Yang M, Li X, Turkbey B, Choyke PL, Yan P., "Prostate segmentation in MR images using discriminant boundary features", IEEE Trans Biomed Eng. 2013 Feb;60(2):47988.

[19] Liyun Yu, Jannick P. Rolland, "Texture-based image enhancement for segmentation performance", Proc. SPIE 3074, Visual Information Processing VI, 82 (July 22, 1997).

[20] Michal Haindl and Stanislav Mikes, "Model-Based Texture Segmentation", Lecture Notes in Computer Science (2004) 306-313.

[21] Shi, J., Malik, J., "Normalized cuts and image segmentation", IEEE Trans. Pattern Anal. Mach. Intell. 22 (2000) 888-905.

[22] Meil, M., Heckerman, D., "An experimental comparison of model-based clustering methods", Mach. Learn. 42 (2001) 9-29.

[23] Andrey, P., Tarroux, P., "Unsupervised segmentation of markov random field modeled textured images using selectionist relaxation", IEEE Transactions on Pattern Analysis and Machine Intelligence 20 (1998) 252-262.

[24] Michal Haindl and Stanislav Mikes, "Colour texture segmentation using modelling approach", ICAPR'05, Pages 484-491.

[25] Nawal Houhou and Xavier Bresson, "Fast Texture Segmentation Model based on the Shape Operator and Active Contour", IEEE Conference on Computer Vision and Pattern Recognition, 2008.

[26] Xiaoli Jiao ; Wen Sheng, "Texture segmentation based on markov random field model and multidirectional mosaics", Proc. SPIE, September 29, 2003.

[27] C. Kervrann IRISA/INRIA, Rennes, F.Heitz, "A Markov random field model-based approach to unsupervised texture segmentation using local and global spatial statistics", IEEE Transactions on Image Processing, Volume 4 Issue 6, June 1995,Page 856-862.

[28] Ning-Yu An, Chi-Man Pun, "Color image segmentation using adaptive color quantization and multiresolution texture characterization”, May 2012.

[29] B. S. Raghavendra, P. Subbanna Bhat, "Contourlet Based Multiresolution Texture Segmentation Using Contextual Hidden Markov Models", Intelligent Information Technology, Lecture Notes in Computer ScienceVolume 3356, 2005, pp 336-343.
[30] S. Arivazhagan, L. Ganesan, "Texture segmentation using wavelet transform", Pattern Recognition Letters, Volume 24, Issue 16, December 2003, Pages 3197-3203.

[31] Charalampidis, D. Kasparis, T., "Wavelet-based rotational invariant roughness features for texture classification and segmentation", Image Processing, IEEE Transactions on Volume: 11 , Issue: 8 , Aug 2002.

[32] Robert M. Haralick, "Statistical and structural approaches to texture", Proc. IEEE, vol. 67, no. 5, pp. 786-804, 1979.

[33] Chen,J., Pappas, T.N. Mojsilovic, A. Rogowitz, "Image segmentation by spatially adaptive color and texture features", Image Processing,2003.ICIP.

[34] Junqing Chen, Pappas, T.N.Mojsilovic, A. Rogowitz, "Perceptually-tuned multiscale color-texture segmentation”, ICIP '04. Page (s): 921 - 924, Volume: 2, 24-27 Oct. 2004.

[35] Junqing Chen; Thrasyvoulos N. Pappas; Alexandra Mojsilovic; Bernice E. Rogowitz, "Perceptual color and spatial texture features for segmentation", 17 June 2003.

[36] Mariana Tsaneva, "Texture Features for Segmentation of Satellite Images", Cybernetics and Information Technologies, Volume 8, No 3, 2008.

[37] Ricardo Dutra da Silva, Rodrigo Minetto, William Robson Schwartz, Helio Pedrini, "Satellite Image Segmentation Using Wavelet Transforms Based on Color and Texture Features", Advances in Visual Computing, Lecture Notes in Computer ScienceVolume 5359, 2008, pp 113-122.

[38] Katayoon Sarafrazi, Mehran Yazdi, Mohammad Javad Abedini, "A New Image Texture Segmentation Based on Contourlet Fractal Features", Arabian Journal for Science and Engineering, December 2013, Volume 38, Issue 12, pp 3437-3449.

[39] Neeraj Sharma, Amit K. Ray, Shiru Sharma, K. K. Shukla Satyajit Pradhan and Lalit M. Aggarwal, "Segmentation and classification of medical images using textureprimitive features Application of BAM-type artificial neural network", Journal of Med. Phys. 2008 Jul-Sep; 33(3): 119-126.

[40] M.Joseph Prakash, Saka.Kezia, Dr.I.Santi Prabha, Dr.V.VijayaKumar, "A New Approach for Texture Segmentation Using Gray Level Textons", International Journal of Signal and Image Processing(IJSIP), vol. 6, no. 3, June 2013, pp.81-89.

[41] M.Joseph Prakash, Dr.V.VijayaKumar, Dr.A.Vinaya Babu, "Morphology Based Technique For Texture Enhancement and Segmentation", Signal \& Image Processing: An International Journal (SIPIJ), Volume 4, Number 1, Feb 2013, pp.49-56.

[42] M.Joseph Prakash, Dr.V.VijayaKumar, "A New Texture Based Segmentation Method to Extract Object from Background", Global Journal of Computer Science and Technology Graphics \& Vision, Volume 12, Issue 15 , Dec 2012, pp. 47-53.

[43] M.Joseph Prakash, Saka.Kezia, Dr.I.Santi Prabha, Dr.V.VijayaKumar, "Innovative Pattern Based Morphological Method for Texture Segmentation-IEEE conference proceedings, Chennai, June 4-6, 2013, pp.1115.

[44] Holschneider, M., Kronland-Martinet, R., Morlet, J. and Tchamitchian, P., "A Real-Time Algorithm for Signal Analysis with the help of the Wavelet Transform", Proc. of Wavelets, Time-Frequency Methods and Phase Space, Springer-Verlag, pp. 289-297, 1989. 


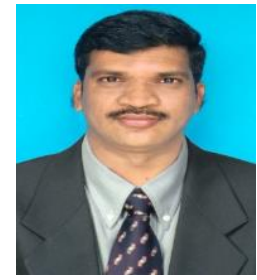

M.Joseph Prakash received the B.Tech Degree from Andhra University in 1996 and received his M.Tech. in Computer Science Engineering from JNTU College of Engineering, JNT University, Kukatpally, Hydera-bad , India in 2003. $\mathrm{He}$ is having nearly 17 years of teaching, research and industrial experience. He taught courses for B.Tech and M.Tech students. He handled various positions Professor, HOD, I/C Principal, Coordinator. $\mathrm{He}$ is currently working as Professor, CSE Dept., School of Engineering and Technology, Guru Nanak Institution Technical Campus (GNITC), Hyderabad, Andhrapradesh, India. He is pursuing his Ph.D from JNT University, Kakinada in Computer Science under the guidance of Dr. V. Vijaya Kumar. He is a life member of Computer Society of India, ISTE, Red Cross Society. He has published 24 papers in various, Inter National Journals and conferences including IEEE.

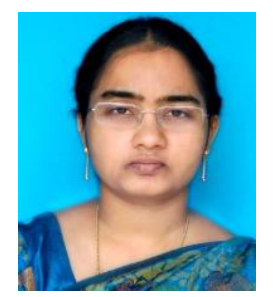

Dr. Kezia. J. M received the B.Tech (ECE) degree from JNTU College of Engineering, JNT University, Kakinada in 2002. She received M.Tech from IIT Madras, India in 2004 and Ph.D from JNTUK in 2013. She is having nearly 11 years of teaching, Research and industrial experience. She is currently working as Professor, Dept of E.C.E, School of Engineering and Technology, Guru Nanak Institution Technical Campus (GNITC), Hyderabad. She is a life member of ISTE, IETE, Red Cross Society. She has presented 20 papers in various Inter National journal and conferences including IEEE.

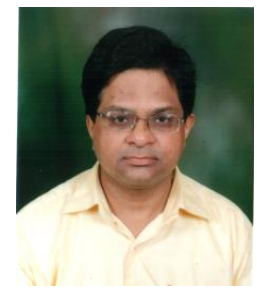

Dr. Vakulabharanam Vijaya Kumar is working as Professor and Dean of CSE \& IT Depts., Anurag Group of Institutions, (Autonomous), Hyderabad. $\mathrm{He}$ received integrated M.S.Engg, in CSE from USSR in 1989. He received his Ph.D. degree in Computer Science from Jawaharlal Nehru Technological University (JNTU), Hyderabad, India in 1998. He has served JNT University for 13 years as Assistant Professor and Associate Professor. He is also acting as director for Centre for Advanced Computational Research (CACR) at AGOI, Hyderabad where research scholars across the state are working. He has received best researcher and best teacher award from JNT University, Kakinada, India. His research interests include Image Processing, Pattern Recognition, Digital Water Marking, Cloud Computing and Image Retrieval Systems. He is the life member of CSI, ISCA, ISTE, IE (I), IETE, ACCS, CRSI, IRS and REDCROSS. He guided 18 research scholars for $\mathrm{Ph} . \mathrm{D}$ and published more than 125 research publications till now in various National, International journals and conferences. He has also established and also acted as a Head, Srinivasa Ramanujan Research Forum (SRRF) at GIET, Rajahmundry, India from May 2006 to April 2013 for promoting research and social activities.

How to cite this paper: Mosiganti Joseph Prakash, Kezia.J.M, V.VijayaKumar,"Morphological Multiscale Stationary Wavelet Transform based Texture Segmentation", IJIGSP, vol.6, no.8, pp.32-39, 2014.DOI: 10.5815/ijigsp.2014.08.05 